\title{
Correction: Design and Characterization of a 52K SNP Chip for Goats
}

\author{
Gwenola Tosser-Klopp, Philippe Bardou, Olivier Bouchez, Cédric Cabau, \\ Richard Crooijmans, Yang Dong, Cécile Donnadieu-Tonon, André Eggen, Henri C. \\ M. Heuven, Saadiah Jamli, Abdullah Johari Jiken, Christophe Klopp, Cynthia T. Lawley, \\ John McEwan, Patrice Martin, Carole R. Moreno, Philippe Mulsant, \\ Ibouniyamine Nabihoudine, Eric Pailhoux, Isabelle Palhière, Rachel Rupp, Julien Sarry, \\ Brian L. Sayre, Aurélie Tircazes, Jun Wang, Wen Wang, Wenguang Zhang, International \\ Goat Genome Consortium
}

We have forgotten to thank our collaborators who provided the Creole samples. The Acknowledgements section should read:

\section{Acknowledgments}

We thank Mohamad Hifzan Rosali (MARDI, Malaysia), Ernie Muneerah Mohd Adhan (NIVB, Malaysia), Ilias Kyriazakis (UTH, Greece), George.C. Fthenakis (UTH, Greece) and Pierre Martin (Capgenes, France) for collecting samples. For the sampling of the Creole goat population, we acknowledge Nathalie Mandonnet, Michel Naves and Rémy Arquet (INRA, Guadeloupe), Régis Alexandre (Chambre d'Agriculture de Guadeloupe), Marylène Madassamy (CABRICOOP) and breeders, as well as the Région Guadeloupe for its financial support. We thank Marcel Amills (UAB, Spain) and Alessio Valentini (Unitus, Italy) for their help in collecting published SNPs. We are grateful to Curt Van Tassell (ARS, USDA, USA), for testing his spacing software on our data and Rabiatul Adawiah Zainal Abidin, (MARDI, Malaysia) for bioinformatics discussions. We acknowledge Thomas Faraut (INRA, France) for helpful discussions. Expression data from "GENIDOV" (coordinated by Eric Pailhoux) and "CHEST-454" (coordinated by Patrice Martin) programmes were used in this publication. We thank Karine Viaud (Illumina Incorporated, USA), for her excellent work on the creation of the beadpool.

We thank Dr. Umi Kalsom Abu Bakar, Project Director and financial support from CMDV (MARDI) and Malaysian Biotechnology Corporation.

The International Goat Genome Consortium (www.goatgenome.org) members include: Paolo Ajmone (Unicatt), Marcel Amills (UAB), Simon Boitard (INRA), Wenbin Chen (BGI), Shifeng Cheng (BGI), Yang Dong (KIZ), Thomas Faraut (INRA), Omar Faruque (BAU), Henri Heuven (UU), Zhao Jinshan (IMAU), Lv Jun (KIZ), Hans Lenstra (UU), Xin Li (KIZ), Xin Liu (BGI), Carole Moreno (INRA), Philippe Mulsant (INRA), Shengkai Pan (BGI), Mario A Poli (INTA), Lichen Ren (KIZ), Su Rui (IMAU), Rachel Rupp (INRA), Magali San Cristobal (INRA), Brian L Sayre (VSU), Bertand Servin (INRA), Peng Shi (KIZ), Chao Song (BGI), Alessandra Stella (PTP), Gwenola Tosser-Klopp (INRA), Alessio Valentini (Unitus), Li Xianglong (HBAU), Xun XU (BGI), Zhang Yanjun (IMAU), Chen Ye (BGI), Jiang Yu (KIZ), Wenguang Zhang (KIZ, IMAU), Bo Zhang (BGI), Shuhong Zhao (HZAU) from the following organizations:

BAU: Bangladesh Agricultural University, Bangladesh

BGI: Bejing Genome Institute, China 
HBAU: Hebei Agricultural University, China

HZAU: Huazhong Agricultural University, China

IMAU: Inner Mongolia Agricultural University, China

INRA: Institut National de la Recherche Agronomique, France

INTA: Instituto Nacional de Tecnología Agropecuaria, Argentina

KIZ: Kunming Institute of Zoology, Chinese Academy of Sciences, China

PTP: Parco Tecnologico Padan, Italy

UAB: Universitat Autònoma de Barcelona, Spain

UU: Utrecht University, The Netherlands

Unicatt: Università Cattolica del S. Cuore, Italy

Unitus: Università della Tuscia, Italy

VSU: Virginia State University, United States of America

\section{Reference}

1. Tosser-Klopp G, Bardou P, Bouchez O, Cabau C, Crooijmans R, Dong Y, et al. (2014) Design and Characterization of a 52K SNP Chip for Goats. PLoS ONE 9(1): e86227. doi:10.1371/journal.pone. 0086227 PMID: 24465974 